Andreas Unger

Fallbeschreibung

Die Anfrage kam in Form einer E-Mail von dem Bruder der Patientin:

«... Führen Sie auch konsiliarische Beurteilungen im Sinne einer Second Opinion bei (komplexen) chronischen Schmerzpatienten durch?

Ich habe eine Schwester, die bei einem Höhensturz vor 13 Jahren eine inkomplette Paraplegie erlitten hat und seither unter einem chronischen Schmerzsyndrom leidet. Leider hat sich ihre Schmerzsymptomatik in den letzten Monaten massiv verschlechtert und kann von der aktuell betreuenden Schmerzklinik nicht mehr zufriedenstellend therapiert werden.

Sehr gerne würde ich sie einer ganzheitlichen Schmerztherapie zuführen.

Welche Möglichkeiten haben wir? Ist eine Beurteilung durch Sie möglich und sinnvoll?»

\section{Vorgeschichte}

Ihre Kindheit erinnert die 37-jährige Patientin als unbeschwert. Neben guten schulischen Leistungen spielte sie Geige sowie Klavier und war im Kunstturnen sowie Tanzunterricht. Nach Primar- und Sekundarschule trat sie in das Lehrerseminar ein. In dieser Zeit erkrankte sie schwer depressiv und erhielt Psychopharmaka und Psychotherapie; es folgten mehrere stationäre Aufenthalte in psychiatrischen Kliniken. Während des letzten Aufenthalts in einer psychiatrischen Klinik stürzte sie sich als 23-Jährige in suizidaler Absicht in einen $10 \mathrm{~m}$ tiefen Liftschacht. Sie hat diesen Sturz mit schwersten Verlet-

\title{
Grenzen überschreiten - Fallvorstellung einer Kombination von Komplementärmedizin mit interventioneller Schmerztherapie
}

zungen und der Folge einer inkompletten Querschnittslähmung überlebt, mit der sie über viele Jahre zu leben gelernt hat. Nach der Rehabilitation war sie fähig, wieder kleine Strecken zu gehen und auch Treppen zu steigen. Sie war in ihrer Wohnung selbstständig sowie mit einem Rollstuhl und einem Personenwagen mobil. Infolge der anhaltenden Schmerzsymptomatik wurde ihr ab 1999 eine volle IV-Rente zugesprochen.

Die Patientin war über mehrere Jahre in Psychotherapie, regelmässig in Physiotherapie und Feldenkrais und seit 2010 wegen der Schmerzen und Obstipation bei einem Chinesen zur Akupunktur und Phytotherapie.

Bis Dezember 2011 war die Patientin autonom. Im Januar 2012 wurde im linken Fuss ein Metallbruch und eine Fistelbildung festgestellt, operativ saniert und eine neue Schmerzpumpe eingebaut. Die nach 4 Monaten Entlastung folgende Mobilisation verlief stark verzögert, und es kam zu neuen Schmerzen und zunehmenden Fehlhaltungen vom Becken aufwärts über die Lendenwirbelsäule (LWS) und Brustwirbelsäule (BWS) zur linken Schulter und schliesslich zu einer Nackenstarre.

Die Schmerzen und Fehlhaltungen wurden als psychogen beurteilt und nahmen trotz regelmässigen ambulanten Therapien und Konsultationen in der sie betreuenden renommierten Schmerzklinik kontinuierlich $\mathrm{zu}$. Nachts konnte sie nicht mehr schlafen, weil sie wegen unerträglicher Schmerzen im gesamten Rückenbereich keine Position mehr zum Liegen fand. 2 Mo- nate vor der stationären Aufnahme brach sie aufgrund von Schmerzen, die keinerlei Bewegung mehr zuliessen, sämtliche Physiotherapien und Feldenkrais ab. Bei anhaltenden Schmerzen zwischen NAS (numerische Analogskala) 8 und 10 von 10 , die keiner Therapie mehr zugänglich waren, resignierte die Patientin, bis ihr Bruder die Initiative ergriff.

\section{Diagnosen}

\section{Mitgebrachte Diagnosen}

Die Liste der mitgebrachten Diagnosen füllte die erste Seite der Arztbriefe:

- Cauda(-equina)-Syndrom mit neuropathischen Schmerzen nach Sturz aus ca. $10 \mathrm{~m}$ Höhe mit

- LWK-5-Berstungsfraktur und komplexer Os-sacrum-Fraktur

- Tibiafraktur, Talus- und Malleolus-medialis-Fraktur rechts

- Calcaneusfraktur links

- Nierenkontusion mit retroperitonealem Hämatom, Pneumothorax links

- Commotio cerebri

- Autonome Dysregulation mit Blasen-, Darm- und Sexualfunktionsstörung

- Chronisches therapierefraktäres neuropathisches Schmerzsyndrom lumbosakral rechtsbetont

- Status nach Implantation einer Medtronic-Pumpe zur intrathekalenSchmerzmittelapplikation am 30.06.01

- Status nach Revision mit Pumpenwechsel am 01.11.01 und am 20.01.04

\section{KARGER}

Fax +497614520714 Information@Karger.com www.karger.com
() 2013 S. Karger GmbH, Freiburg

Accessible online at: www.karger.com/szg
Andreas Unger

Leitender Arzt Integrative Schmerztherapie

Aeskulap-Klinik Brunnen

Gersauerstrasse 8, 6440 Brunnen, Schweiz

andreas.unger@aeskulap.com www.aeskulap-klinik.ch 
- Status nach Revision des intrathekalen Katheters mit Punktion Th12/L1, Katheterspitze Höhe Th7/8 und Schmerzpumpenwechsel Medtronic-Pumpe $40 \mathrm{ml}$ am 21.09.06

- Status nach Metallentfernung in LWS und Becken

- Status nach Anlage eines Anus praeter 2009 und Rückverlegung 2010

- Status nach rasch aszendierendem Querschnittssyndrom seit 15.09.06, sensibel links sub Th6, rechts sub L1

- Katheterspitzengranulom auf Höhe BWK6

- Postoperatives Liquorverlustsyndrom

- Intermittierende Schwäche des rechten Beins, neurologisch nicht zuordenbar

\section{Erste Konsultation}

Zur ersten Konsultation erschien die Patientin von ihrem Bruder begleitet im Rollstuhl hängend, auf seltsame Art nach rechts verdreht und geneigt. Die Halswirbelsäule (HWS) war gegenläufig verdreht und geneigt, der Kopf in starrer Haltung, die linke Schulter hochstehend und der linke Arm in Adduktion am Körper fixiert. Schon der Blickkontakt schien für sie mit einer grossen Anstrengung verbunden. Den Transfer vom Rollstuhl auf die Liege führte sie erstaunlicherweise selbstständig durch und lehnte angebotene Hilfe ab. Aufgrund starker Schmerzen in einer starken Fehlhaltung mit einer Beugekontraktur im Becken konnte sie sich nur mit dem rechten Arm, dem Körper und teilweise dem Kopf abstützend in einer kriechenden Bewegung aus dem Rollstuhl auf die Liege bewegen. Eine Aufrichtung der Wirbelsäule war weder im Sitzen noch im «Stehen» oder Liegen, weder aktiv noch passiv möglich; eine Bewegung der LWS war lediglich in die schon bestehende starke Rechtsseitneigung und Rotation, die der Hüften in eine weitere Flexion möglich.
Die HWS, der zervikothorakale Übergang und die linke Schulter waren wie spastisch in einer Fehlhaltung fixiert; bei nahezu vollkommen aufgehobener Beweglichkeit waren manualmedizinisch keine funktionellen Untersuchungen möglich. Die Ursachen der Schmerzen schienen im Bereich der Wirbelsäule zu liegen; weiter liessen sie sich zunächst nicht eingrenzen. Die Paresen der Beine und des Rumpfs waren, soweit untersuchbar, Kraftgrad 4 von 5 und schienen zumindest für den vordergründigen Schmerz nachrangig.

Medikation: Intrathekale Schmerzmittelapplikation von Bupivacain, Hydromorphon und Clonidin über eine Medtronic-Pumpe mit einer Basis und maximal 10 Boli täglich in einem Intervall von nicht unter $2 \mathrm{~h}$. Dazu bei Bedarf Ketamin Nasenspray $25 \mathrm{mg} / \mathrm{ml}$, das sie (bei fraglicher Wirkung) maximal ausnutzte.

An weiteren Medikamenten wurden Sirdalud $4 \mathrm{mg} 2 \times 1$ täglich zur Muskelentspannung, Rivotril $2 \mathrm{mg}$ $2 \times 1$ täglich gegen den neuropathischen Schmerz, Detrusitol $4 \mathrm{mg} 2 \times 1$ täglich sowie Bactrim und Monuril alle 14 Tage im Wechsel wegen rezidivierender Harnwegsinfekte bei Blasenfunktionsstörung eingenommen. Hinzu kamen bei Bedarf Buscopan, Dafalgan und Tranxilium in hohen Dosen.

\section{Ergänzende Diagnosen}

Unsere ergänzenden Diagnosen nach der ersten Konsultation:

- Myofasziales Schmerzsyndrom bei Funktionsstörungen der Kopfgelenke und des zervikothorakalen Übergangs mit Kopfschmerzen, Tinnitus und Sehstörungen

- Posttraumatisches myofasziales Schmerzsyndrom dorsolumbosakral

- Schlafstörung - schmerzbedingt maximal $2 \mathrm{~h} / \mathrm{Nacht}$

- Schmerzbedingte Anpassungsstörung

- Koprostase

Bereits beim ersten Kontakt wurde deutlich, dass in diesem Fall eine ambulante Behandlung sicher nicht zum Ziel führen würde. Vor einer stationären Aufnahme in der AeskulapKlinik benötigten wir eine weitere Diagnostik.

\section{Prästationäre Abklärung}

Eine neurologische Abklärung erbrachte keine nicht schon bekannten oder weiterführenden Befunde. Mit einer Magnetresonanztomographie (MRT) der Wirbelsäule konnten neu aufgetretene «strukturelle» Befunde wie Bandscheibenvorfälle, Spinalkanalstenosen oder Frakturen ausgeschlossen werden. Indikationen für ein operatives Vorgehen oder andere schulmedizinische Therapiemöglichkeiten bestanden also nicht.

$\mathrm{Da}$ integrative Medizin für uns nicht nur schul- und komplementärmedizinisch, sondern ganzheitlich auch die körperlichen und psychischen Anteile von Schmerzen behandelt, sollte ein psychiatrisches Konsil Aufschluss über mögliche psychosomatische Therapieansätze geben. Hinweise auf eine somatoforme Schmerzstörung ergaben sich dabei nicht.

Bei einer osteopathischen Probebehandlung schien die auf den ersten Blick spastisch anmutende Fehlhaltung manualmedizinischen und osteopathischen Techniken zugänglich zu sein. Von umfassenden komplementärmedizinischen Untersuchungen erhofften wir uns weitere Therapieansätze.

\section{Komplementärmedizinische Untersuchungsergebnisse}

Das schulmedizinische Labor war bis auf ein deutlich erniedrigtes Vitamin D3 (11 $\mu \mathrm{g} / \mathrm{l})$ und erhöhtes Homocystein $(28,6 \mu \mathrm{mol} / \mathrm{l})$ unauffällig. Bei regelmässiger Katheterisierung und chronisch rezidivierenden Harnwegsinfekten war Escherichia coli im Urin nachweisbar. 
Die Mikronährstoffanalyse ergab Hämatokrit-korreliert niedriges Magnesium und stark erniedrigtes Zink und Selen. Die mit 1,86 mg/g Kreatinin erhöhte Methylmalonsäure im Urin werteten wir als Hinweis auf nitrosativen Stress. Der FRAS(Free Radical Analytic System)-Test zeigte im d-Rom(Diacron Reactive Oxygen Metabolites)-Test mit 446 CARR U einen hohen oxidativen Stress und im BAP(biologisches antioxidatives Potenzial)-Test mit $3586 \mu \mathrm{mol} / \mathrm{l}$ ein gutes antioxidatives Potenzial, das bei der Raucherin aber nicht in der Lage war, den hohen oxidativen Stress zu kompensieren. In der BEV (Bioelektronik nach Vincent) fanden sich ebenfalls mit der Übersäuerung im Blut und Urin als Hinweis auf eine kompensatorische Säureausscheidung sowie mit den vermehrten Sauerstoffradikalen im Blut Zeichen für eine intrazelluläre mesenchymale Azidose und für oxidativen Stress. Der Spengler-Test wies neben der Gewebebelastung $(\mathrm{OM}+++)$ auch auf Störfelder hin (D +++, Dx +++), die sich differenzierter auch in der Thermoregulationsdiagnostik (TRD) wiederfanden. Die TRD und das Indikan im Uricolor-Test gaben Hinweise auf den Darm; der Florastatus zeigte einen erhöhten pH-Wert und eine reduzierte Säuerungsflora. Die zur Fokussuche erstellte Panoramaschichtaufnahme war bis auf retinierte Weisheitszähne $(18,28)$ unauffällig.

Daraus ergaben sich für uns mehrere Therapieansätze. Zur Schmerzreduktion, zur Entzündungshemmung, zur Neutralisierung freier Radikale und vegetativ ausgleichend verordneten wir Procain-Basen-Infusionen sowie Hochdosis-VitaminC- und Vitamin-B12-Infusionen und substituierten Vitamine und Spurenelemente. Entsprechend der Symptomatik behandelten wir mit Neuraltherapie, Akupunktur, Osteopathie sowie C-Bogen-gesteuerten Interventionen. Physiotherapie, Feldenkrais, Neurologisches Integrationssystem (NIS), Ozon-Therapie, Hydro-Colon-Thera- pie und probiotische Therapie sowie begleitende Psychotherapie sollten die Behandlung unterstützen.

\section{Die ersten Tage}

Neben der starken Schmerzabwehrhaltung der HWS, BWS und LWS litt die Patientin unter Kopfschmerzen, Tinnitus und extremen schmerzbedingten Schlafstörungen und konnte aufgrund ausgeprägter Sehstörungen nur noch einige Sekunden lesen. Wie tagsüber im Rollstuhl hängend, verbrachte sie auch die Nächte in extremer Verkrümmung und Verdrehung in halb aufrechter Lage. Hohe Dosen an Opioiden, Muskelrelaxanzien und Antikonvulsiva hatte sie schon in ihrer Vorgeschichte als wirkungslos erfahren; dennoch versuchten wir, in den Nächten mit Hydromorphoninfusionen und Amitriptylin in schmerzdistanzierender Dosis wenigstens etwas Schlaf und Erholung zu erreichen.

Da die Patientin weder liegen noch stehen konnte, wurden die abgebildeten Röntgenaufnahmen im Rollstuhl angefertigt. Sie zeigen die starken Fehlhaltungen und Verdrehungen vor allem im Bereich der HWS und LWS (Abb. 1-6).

\section{Osteopathische Behandlung in drei Phasen}

Die Behandlung wurde durch einen in Osteopathie ausgebildeten Arzt mit Schwerpunkt Craniosacrale Therapie, eine Physiotherapeutin und eine Krankenschwester in engem Kontakt durchgeführt.

In der ersten Phase konnte die Patientin den Schmerz nicht differenzieren; sie beschrieb ihn wie einen «zusammengezogenen Eisklumpen Schmerz». Sie lernte zunächst, einmal ihren Körper so wahrzunehmen, dass Veränderung möglich wurde und dass wir, nachdem ihre Schmerzen von ihren behandelnden Ärzten über

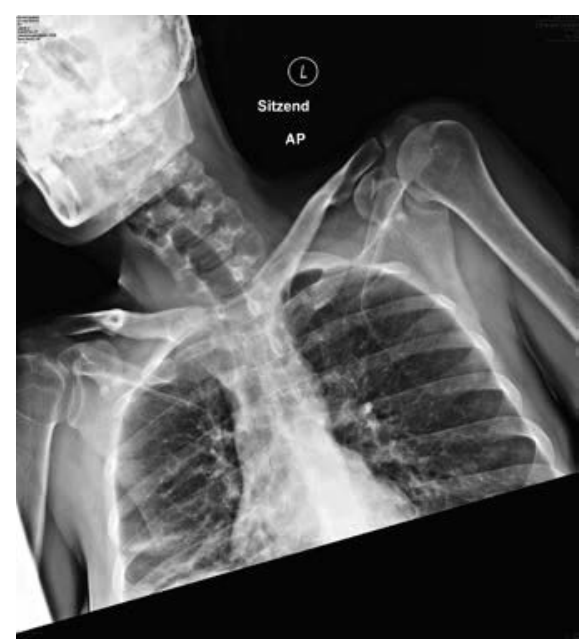

Abb. 1. HWS anterior-posterior, sitzend.

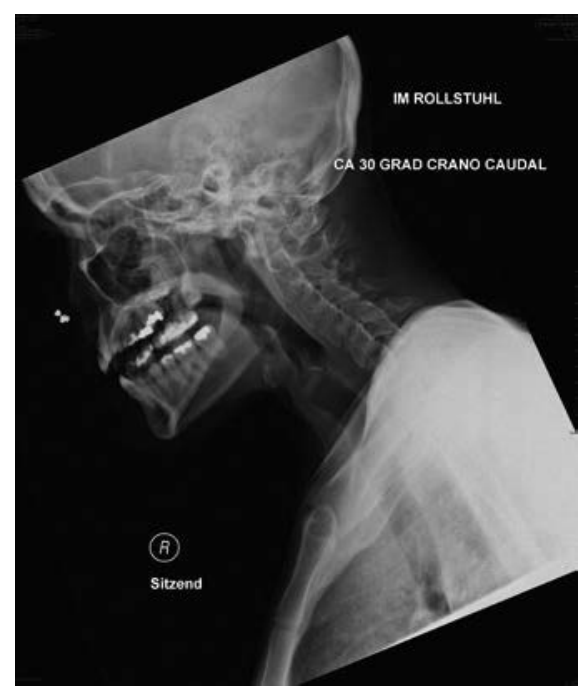

Abb. 2. HWS seitlich, im Rollstuhl, ca. $30^{\circ}$ craniocaudal.

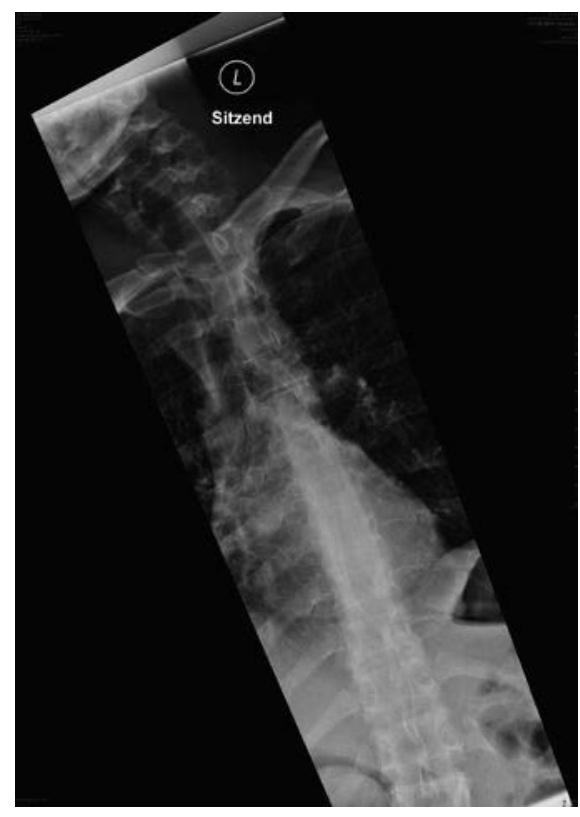

Abb. 3. BWS anterior-posterior, sitzend. 


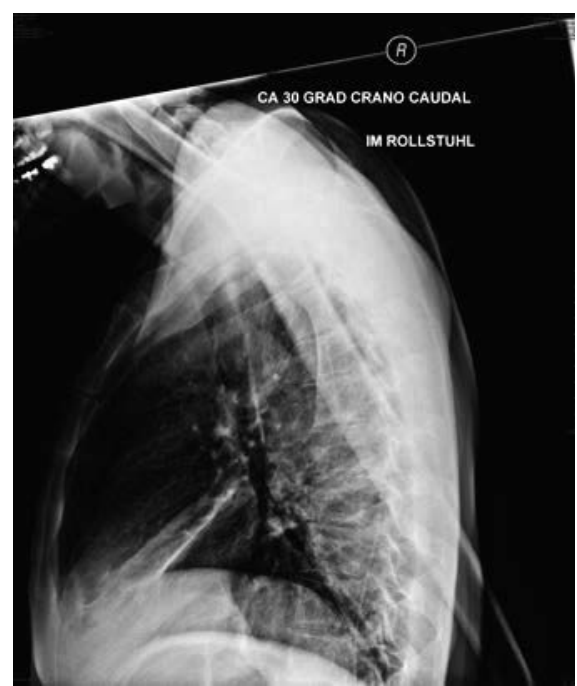

Abb. 4. BWS seitlich, im Rollstuhl, ca. $30^{\circ}$ craniocaudal.

Jahre als psychogen gedeutet wurden, sie mit ihrer Persönlichkeit akzeptierten.

Nach Reflexakupunktur nach Felix Mann konnten im Liegen bereits nach der ersten Behandlung kleine Rotationsbewegungen im Bereich der HWS erreicht werden. Mit vorsichtiger Mobilisierung und Unwinding der oberen HWS, paravertebralem myofaszialen Release der BWS, spinaler Duraarbeit und faszialer Entlastung, rhythmischer Begleitung sowie Synchronisierung konnten eine Schmerzreduktion und Reduktion des Gewebetonus erreicht werden. An ihrer Fehlhaltung hatte sich damit noch nicht viel verändert, aber durch den intensiven Kontakt entstand ein anderes Körpergefühl, ein Annehmen des zuvor abgelehnten, aufgrund der Schmerzen nur noch als Ballast empfundenen Körpers, d.h. ein Wahrnehmen von anderen Sensationen als Schmerz, und eine Bereitschaft, Veränderungen zuzulassen. Der Hauptschmerz liess sich jetzt im Bereich der mittleren LWS links in Höhe L3/4 lokalisieren, woraus die Idee der Facetteninfiltration entstand.

\section{Die Facetteninfiltration}

Der erste Eingriff unter dem C-Bogen fand unter aussergewöhnlichen Bedingungen statt. Die Patientin kroch - sich mit dem Kopf abstützend - vom Rollstuhl auf die Liege, wo wir bei in Flexion kontrakter Hüfte mit allen zur Verfügung stehenden Mitteln eine Mischung aus kniender und liegender Lagerung erreichten, in der die immer noch stark von Schmerzen geplagte Patientin für wenige Minuten für die Intervention unter dem C-Bogen ruhig «liegen» konnte. Die bis dahin durchgeführten MRTUntersuchungen waren in Narkose durchgeführt worden. Nach Infiltration der Facettengelenke L2/3 und L3/4 links mit Bupivacain und Prednisolon konnte sich die Patientin dann nach Monaten erstmals wieder aufrichten.

Wir sahen also zunächst in der Facetteninfiltration den Schlüssel und überlegten, wie sich eine Verödung der Facetten mit Radiofrequenztherapie auf die Programmierung der Medtronic-Pumpe auswirken würde.

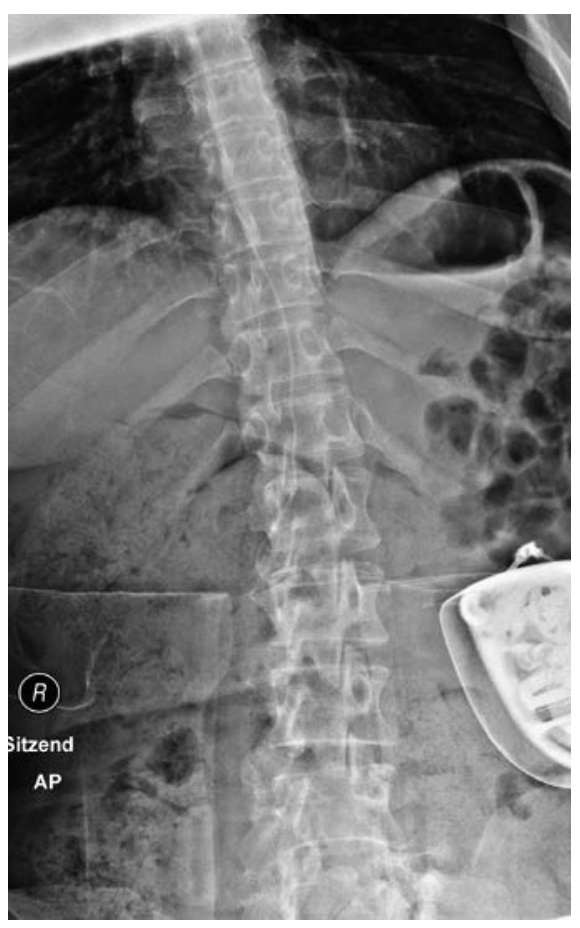

Abb. 5. LWS, anterior-posterior, sitzend.

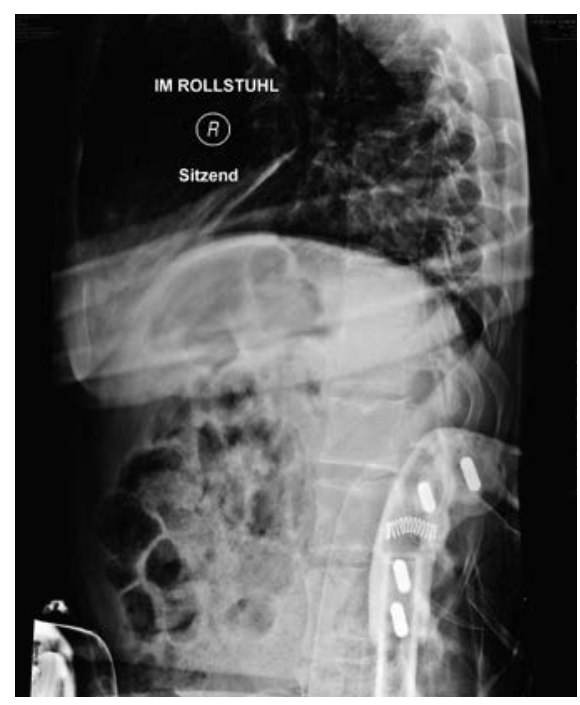

Abb. 6. LWS seitlich, sitzend, im Rollstuhl.

In der jetzt folgenden zweiten Phase wurden viszerale Techniken für Dickdarm, Dünndarm und Leber und koordinative Übungen für die tiefe Bauchmuskulatur und zur Stabilisierung der LWS eingesetzt. Auch auf Akupunktur, Neuraltherapie und myofasziales Release reagierte sie sehr gut. Häufig verliess sie die Therapien nicht mehr im Rollstuhl sitzend, sondern nahezu aufrecht gehend. Die medikamentöse Therapie wurde um Cannabis-Tinktur als Co-Analgetikum und Muskelrelaxans zur Stimmungsaufhellung und Appetitsteigerung ergänzt.

9 Tage später wurde eine weitere Facetteninfiltration L2/3, L3/4 und L4/5 links mit Bupivacain und Betamethason bereits in entspannter Bauchlage durchgeführt.

\section{Der Darm}

Eine gestörte Darmfunktion hat Auswirkungen auf den ganzen Körper; die Hydro-Colon-Therapie ist eines der klassischen Therapieverfahren bei diesen Störungen. In diesem Fall konnte mit den Hydro-ColonTherapien aufgrund der massiven 
Koprostase mit bis in das Colon ascendens reichenden Kotsteinen wenig gelöst werden. Wie sich herausstellte, hatte die Patientin regelmässig über mehrere Wochen keinen Stuhl, da die digitale Ausräumung mit Zertrümmerung der Kotsteine mit starken Schmerzen verbunden war und aufgrund einer dabei entstandenen Verletzung bereits $\mathrm{zu}$ einer zeitweiligen Anlage eines Anus praeter geführt hatte. Nachdem verschiedene pflanzliche Laxanzien und Einläufe, Akupunktur und Movicol nicht zielführend waren, haben wir uns entschieden, die Darmentleerung mit dem Einsatz eines Präparates zur Vorbereitung von Koloskopien (Moviprep) zu erzwingen. Danach sollte mit einem Management des Stuhlgangs mit Laxanzien und selbstständig durchgeführten Einläufen (Peristeen) eine erneute Koprostase vermieden werden. Vor einer Radiofrequenztherapie der lumbalen Facettengelenke wollten wir so zunächst den Darm als eine über segmentale Verknüpfungen zur LWS mögliche Ursache für die lumbalen Schmerzen und Fehlhaltungen behandeln.

Dementsprechend standen in der dritten Phase der Behandlung osteopathisch die Darmfunktion und vegetative Regulation über Unterstützung und Entrainment der autonomen Rhythmen im Vordergrund. Die Patientin kam mittlerweile ohne Rollstuhl $\mathrm{zu}$ den Behandlungen.

\section{Eine starke Persönlichkeit}

Kein Schicksal einer Patientin hat uns je annähernd so berührt wie dieses. Sie war nicht dazu bereit, das Rauchen und den Coca-Cola-Konsum aufzugeben, und lehnte auch unser psychotherapeutisches Angebot ab. Von monatelangen schlaflosen Nächten müde und erschöpft verteidigte sie kämpferisch ihre Grenzen und Integrität. Ohne sie ernst zu nehmen bzw. mit ihrer Persönlichkeit und ihren Grenzen anzunehmen, hätte es keine heilsame therapeutische Beziehung gegeben - so eindeutig wurde uns das nur durch sie gezeigt. «Den Patienten da abholen, wo er steht», sagt sich manchmal einfacher, als es ist.

\section{Das Ergebnis}

Die Patientin steht und geht wieder (Abb. 7); für längere Strecken benutzt sie wie in den Jahren zuvor ihren Roll-

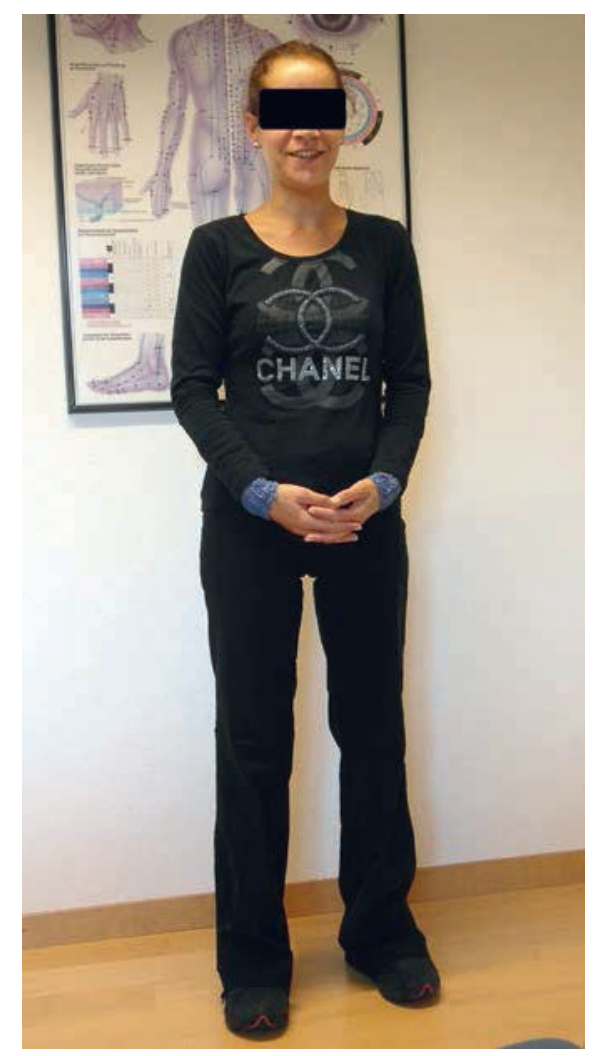

Abb. 7. Nach dem Aufenthalt in der AeskulapKlinik. stuhl. Mit der Regulation des Stuhlgangs erübrigten sich weitere Interventionen an der LWS. Wir sehen sie nach einer kurzen Pause ein- bis zweimal wöchentlich ambulant, um das Erreichte zu stabilisieren und in der Hoffnung, zu gegebener Zeit auch die verbliebenen Schmerzen noch zu verbessern. Dass sie nach über 6 Jahren unter unserer Therapie wieder ihre Mens bekam, hatte uns alle überrascht. Man kann sich fragen, an welchen «Rädchen» wir da eigentlich gedreht haben.

\section{Fazit}

Wir alle haben aus diesem Fall viel gelernt. Es kann sich lohnen, einen Weg zu gehen, von dem man am Anfang noch nicht weiss, wo er hinführt, Grenzen zu überschreiten, «aus dem Bauch heraus» zu handeln und dabei kritisch zu überprüfen und zu hinterfragen.

In den meisten Fällen führen mehrere Wege zum Ziel, und häufig entscheiden die Einstellung des Patienten oder die Ausbildung des behandelnden Arztes über operatives oder konservatives, schulmedizinisches oder komplementärmedizinisches Vorgehen. In dem hier dargestellten Fall ohne operative Alternative erreichte die schulmedizinische Schmerztherapie ihre Grenzen und das Potenzial des Konzepts einer integrativen Schmerztherapie mit der Verbindung klassischer komplementärmedizinischer Therapien wie Osteopathie, Neuraltherapie, Akupunktur und orthomolekulare Medizin mit interventionellen Verfahren in einem empathischen, gut strukturiert zusammenarbeitenden Team wird umfänglich deutlich. 